\title{
Qualidade da água do reservatório de Nossa Senhora das Dores através do índice de qualidade da água de reservatório
}

\author{
Quality of water reservoir of Nossa Senhora das Dores through the index of reservoir water \\ quality
}

\author{
A. F. Silva ${ }^{*}$; C. A. B. Garcia ${ }^{2}$; S. S. L. Costa; N. C. M. Santos; J. M. Silva; H. L. \\ Garcia

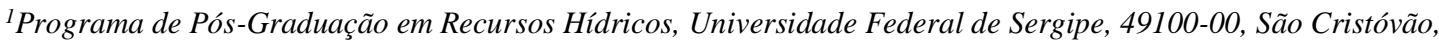 \\ Sergipe, Brasil \\ ${ }^{2}$ Laboratório de Química Ambiental, Universidade Federal de Sergipe, 49100-00, São Cristóvão, Sergipe, Brasil
}

*anamalia@outlook.com

(Recebido em 30 de maio de 2017; aceito em 28 de setembro de 2017)

\begin{abstract}
O atual estudo objetivou avaliar a qualidade sazonal da água do Açude Público, reservatório localizado no município de Nossa Senhora das Dores - Sergipe. Este pertence à sub-bacia do rio Siriri, encontrando-se nas coordenadas $10^{\circ} 29^{\prime} 31,32^{\prime}$ 'S $-37^{\circ} 11^{\prime} 11,83^{\prime}$ 'O . A etapa de monitoramento realizou-se no período de 2014 a 2016, com a amostragem nos períodos seco e chuvoso. As medidas de temperatura da água e profundidade foram realizadas in situ, os demais parâmetros foram armazenados, refrigerados e levados ao laboratório para as análises. Quanto ao Índice de Qualidade de Água de Reservatório - IQAR, o Açude Público possui Classe IV- criticamente degradado a poluído, tanto para o período seco quanto para o período chuvoso. Em relação a comparação com a legislação vigente, Resolução CONAMA 357/05, o reservatório encontra-se impróprio para o uso. Através das análises, conclui-se que há indícios de que o reservatório encontra-se eutrofizado e fora dos padrões da legislação vigente.

Palavras-chave: Sub-bacia do rio Siriri, Sazonalidade, Qualidade da água.
\end{abstract}

This study aimed to evaluate the seasonal quality of the water from the Public Water Reservoir located in the municipality of Nossa Senhora das Dores - Sergipe. This reservoir belongs to the sub-basin of the Siriri river, located in the coordinates $10^{\circ} 29^{\prime} 31,32$ "S - 37 ${ }^{\circ} 11^{\prime} 11,83^{\prime \prime O}$. The monitoring activities were performed in the period between 2014 and 2016. The water samples were collected in the dry and rainy periods and water temperature and depth of sampling were measured in situ. The samples were stored in 3 aliquots, refrigerated and taken to the laboratory for analysis. As for the Water Quality Index of Reservoir - IQAR, the Public Water has Class IV - critically degraded to polluted, both for the dry period and for the rainy season. In comparison with the current legislation, Resolution CONAMA 357/05, the Public Water is improper for the use. Through the analyzes, it was observed that the reservoir is eutrophic and outside the standards of the current legislation.

Keywords: RWQI, water quality, seasonality

\section{INTRODUÇÃO}

Reservatórios são formados pelo represamento de um rio ou riacho, ou através da construção de vales em um local impermeável com fornecimento de um canal, chamado de represas. Apesar de receberem o nome de lagos artificiais, as características existentes entre reservatórios e lagos naturais os diferem em relação ao funcionamento do seu ecossistema [1].

Com o objetivo de solucionar os impactos ocasionados pelo baixo índice de precipitação, a construção de reservatórios é de fundamental importância para suprir as demandas existentes pela população que reside na região semiárida brasileira, além de serem vistos como uma tecnologia acessível [2].

Contudo, essas construções trazem consigo danos inerentes ao seu novo sistema hídrico. Segundo Ferreira e Cunha (2013) [3], a transformação de um ambiente lótico em lêntico modifica o regime térmico dos fluxos, além de provocar mudanças nas condições químicas e biológicas do 
ambiente, esses impactos perpassam do âmbito ambiental e chegam a níveis sociais, econômicos, políticos e culturais.

A avaliação da qualidade da água nesses ambientes torna-se necessária para inferir os fatores que ocasionam o processo de deterioração ambiental ocasionado tanto por atividades antrópicas como naturais. Considerando que sistemas lênticos, tais como lagos, reservatórios e lagoas são mais vulneráveis à poluição, os programas de monitoramento da qualidade da água dão suporte aos gestores da região hidrográfica para tomada de decisão através do prognóstico da situação hídrica [4]. Essa análise se dá pela coleta, análise e interpretação dos dados físico-químicos e microbiológicos do sistema aquático de acordo com a sazonalidade.

Diante desse contexto, indicadores ambientais são ferramentas de fundamental relevância para gestão ambiental de corpos hídricos. Especificamente, o Índice de Qualidade de Água de Reservatórios - IQAR, proposto pelo Instituto Ambiental do Paraná, caracteriza os reservatórios em diferentes classes de acordo com o grau de degradação da qualidade de suas águas [5].

O município de Nossa Senhora das Dores, ao longo dos anos, vem expandindo sua ocupação no entorno do reservatório. A realidade sanitária do município não difere de outras cidades brasileiras que de maneira geral descarta seus efluentes domésticos diretamente nos rios. Segundo o ATLAS de Saneamento básico de 2014 [6], o município possui um percentual de água tratada de $100 \%$, ausência de uma rede coletora de esgoto, além de ter como destino final dos resíduos sólidos, os vazadouros a céu aberto (lixão).

Atendendo as questões apresentadas, o seguinte trabalho objetivou avaliar a qualidade da água do reservatório de Nossa Senhora das Dores através do Índice de Qualidade de Água de Reservatório, o IQAR.

\section{MATERIAL E MÉTODOS}

\section{1 Área de estudo}

O reservatório em estudo, Açude Público, localiza-se no município de Nossa Senhora das Dores, região central do Estado de Sergipe, pertencente à Bacia Hidrográfica do Rio Japaratuba, encontrando-se nas seguintes coordenadas $\left(10^{\circ} 29^{\prime} 31,32\right.$ '’S - 37'11'11,83’'O) (Figura 1).

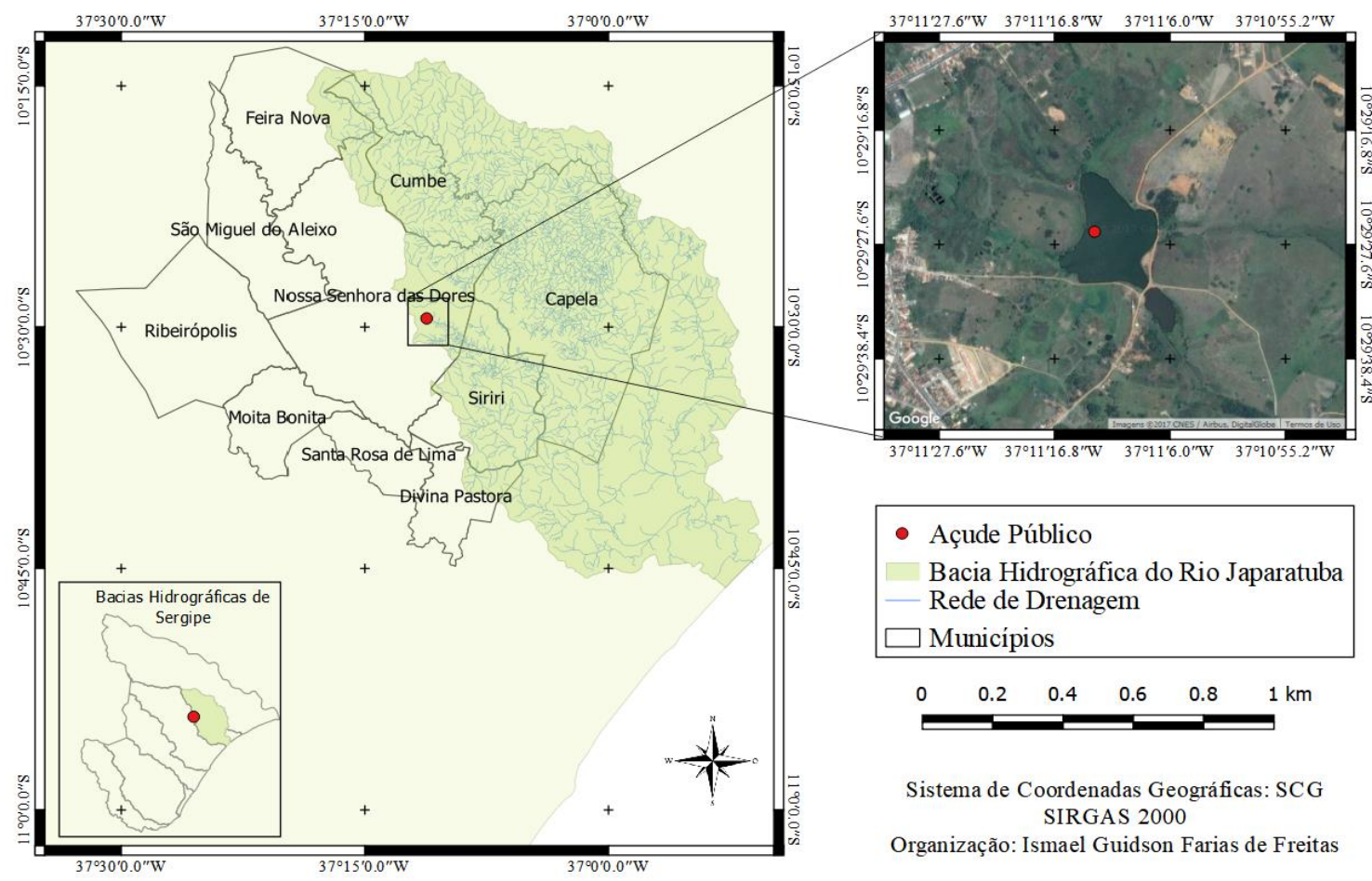

Figura 1: Localização da área de estudo [7] 
O reservatório limita-se ao norte com os municípios de Feira Nova e Cumbe, a leste com Capela e Siriri, a sul com Divina Pastora, Santa Rosa de Lima e Moita Bonita e a oeste com Ribeirópolis e São Miguel do Aleixo [7].

Na fundação do município de Nossa Senhora das Dores, o Açude Público destinava-se a diversos fins, como higiene, abastecimento, etc. Atualmente, como o sistema de abastecimento de água da cidade é realizado pela Companhia de Saneamento de Sergipe - DESO, a utilidade do reservatório restringi-se apenas como um ambiente paisagístico, à custa da falta de saneamento básico.

Em relação à classificação climática de Köppen-Geiger, o clima da região é do tipo megatérmico seco e sub-úmido com transição para semiárido, temperatura média anual de $24,6^{\circ} \mathrm{C}$, precipitação pluviométrica média no ano de 1.056,4 mm e período chuvoso de março a agosto [8].

\subsection{Procedimentos de análise}

Os dados utilizados neste trabalho foram obtidos através das campanhas realizadas no período de 2014 a 2016, totalizando 10 coletas, contemplando a sazonalidade em todos os anos. As amostras foram coletadas em três pontos distintos, distribuídos entre a margem e o centro do reservatório, obtendo-se como resultado a media entre eles.

Os parâmetros analisados seguiram o protocolo de coleta, conservação e análise físico-química e biológica de acordo metodologia descrita pelo Standard Methods for the Examination of Water and Wastewater [9].

As determinações de temperatura e profundidade média foram inferidas in situ. Para as demais análises, as amostras foram coletadas em recipientes de polietileno com capacidade de $0,5 \mathrm{~L}$ cada. Para as amostras de oxigênio dissolvido foi utilizado um frasco de winkler. Para conservação da amostra pra análise de Oxigênio Dissolvido foram adicionados $2 \mathrm{~mL}$ de sulfato manganoso (reagente 1) e $2 \mathrm{~mL}$ de iodeto azida (reagente 2) no momento da coleta. Todos os frascos foram devidamente armazenados sob refrigeração e encaminhados ao Laboratório de Química Analítica Ambiental - LQA, do departamento de Química da UFS e ao Instituto Tecnológico e de Pesquisas do Estado de Sergipe - ITPS para as determinações analíticas.

Para o cálculo do Índice da Qualidade de Água de Reservatórios (equação 1), as variáveis selecionadas receberam pesos distintos em função de seus diferentes níveis de importância na avaliação da qualidade da água [5]. Esses parâmetros, juntamente com seus respectivos pesos, são descritos como déficit de oxigênio dissolvido (17\%), clorofila $a$ (15\%), fósforo total (12\%), profundidade Secchi (12\%), demanda química de oxigênio (12\%), tempo de residência (10\%), nitrogênio inorgânico total (8\%), número de cianobactérias (8\%) e profundidade média (6\%) [5].

$$
I Q A R=\frac{\sum\left(q_{i} w_{i}\right)}{\sum w_{i}} \quad \text { (Equação 1) }
$$

Onde:

IQAR - Índice de Qualidade da Água de Reservatórios;

$q_{i}$ - classe de qualidade de água em relação a variável "i", que pode variar de 1 a 6.

$w_{i}$ - pesos calculados para as variáveis "i".

As seis classes de qualidade de água de acordo com o Instituto Ambiental do Paraná - IAP [5] estão dispostas na Tabela 1. 
Tabela 1: Classes de qualidade da Água

\begin{tabular}{cc}
\hline Valor do IQAR & Qualificação \\
\hline $\mathbf{0} \leq \mathbf{I Q A R} \leq \mathbf{1 , 5}$ & Não impactado a muito pouco degradado \\
$\mathbf{1 , 5 1} \leq \mathbf{I Q A R} \leq \mathbf{2 , 5}$ & Pouco degradado \\
$\mathbf{2 , 5 1} \leq \mathbf{I Q A R} \leq \mathbf{3 , 5}$ & Moderadamente degradado \\
$\mathbf{3 , 5 1} \leq \mathbf{I Q A R} \leq \mathbf{4 , 5}$ & Criticamente degradado a polúído \\
$\mathbf{4 , 5 1} \leq \mathbf{I Q A R} \leq \mathbf{5 , 5}$ & Muito poluído \\
$\mathbf{I Q A R}>\mathbf{5 , 5 1}$ & Extremamente poluído \\
\hline
\end{tabular}

Conforme recomendado na literatura, quando não se dispõe de um parâmetro, é possível calcular o índice desconsiderando-o, bem como seu peso [10]. Assim sendo, o número de cianobactérias e a profundidade pelo Disco de Secchi foram desprezados.

Outra modificação feita para nos parâmetros que compõem o cálculo do IQAR foi substituir a análise da demanda química de oxigênio (DQO) pela do carbono orgânico dissolvido (COD), devido problemas diversos. Alternativa plausível, visto que, a determinação do carbono orgânico total pode fornecer evidências de contaminação por compostos orgânicos [11], e, conforme Metcalf e Eddy (2016) [12], o COT pode ser utilizado para caracterizar a poluição de um esgoto. Além disso, em estudos comparativos sobre alternativas de determinação da DQO pelo COT, Barcella (2016) [13], propôs a substituição da DQO pelo COT em águas de rios por se tratar de um método equivalente e oportuno no conceito da Química Verde.

\section{RESULTADOS E DISCUSSÃO}

Tratando-se de ambientes lênticos, como os reservatórios, os valores do IQAR (Tabela 2) possibilitam caracterizar em que condições ambientais encontram-se o corpo hídrico. Dessa forma, a inferência de alterações ambientais pode ser atribuída às atividades ocorridas em seu entorno.

Tabela 2: Resultado do IQAR

\begin{tabular}{cccc}
\hline Campanha & Período & IQAR & $\begin{array}{c}\text { Classe da } \\
\text { qualidade da } \\
\text { água com base } \\
\text { no IQAR }\end{array}$ \\
\hline C1 $-\mathbf{0 2} / \mathbf{2 0 1 4}$ & Seco & 5,16 & V \\
C2 $-\mathbf{0 5 / 2 0 1 4}$ & Chuvoso & 3,67 & IV \\
C3 $-\mathbf{0 8 / 2 0 1 4}$ & Chuvoso & 3,67 & IV \\
C4 $-\mathbf{1 1 / 2 0 1 4}$ & Seco & 3,90 & IV \\
C5 $-\mathbf{0 2} / \mathbf{2 0 1 5}$ & Seco & 3,16 & III \\
C6 $-\mathbf{0 7 / 2 0 1 5}$ & Chuvoso & 4,01 & IV \\
C7 $-\mathbf{1 1 / 2 0 1 5}$ & Seco & 4,06 & IV \\
C8 $-\mathbf{0 1 / 2 0 1 6}$ & Seco & 3,23 & III \\
C9 $-\mathbf{0 5 / 2 0 1 6}$ & Chuvoso & 4,18 & IV \\
C10 $-\mathbf{1 0 / 2 0 1 6}$ & Seco & 4,18 & IV \\
\hline
\end{tabular}

Os resultados do IQAR apresentados para as campanhas de monitoramento realizadas no reservatório de Nossa Senhora das Dores, no período de 2014 a 2016, demonstram que o intervalo de valores do IQAR variou entre 3,16 e 5,16, com classificação de moderadamente degradado (menor valor) e muito poluído (maior valor). 
Observa-se ainda que, entre 3,67 e 4,18, correspondentes a Classe IV- criticamente degradado a poluído, os valores do IQAR mantiveram-se em predominância durante o período de amostragem, com cerca de $70 \%$ de ocorrência, apresentando um resultado elevado, e, portanto, negativo para a qualidade da água do reservatório.

Conforme a definição das classes proposta pelo Instituto Ambiental do Paraná, sistemas hídricos classificados com a Classe IV são caracterizados com acentuada entrada de matéria orgânica e com aporte considerável de cargas de nutrientes, indicando uma ocorrência de eutrofização, além do desenvolvimento maciço de populações de algas e baixa transparência das águas. Ainda na classe IV é possível a ocorrência de mortandade de peixes em determinados períodos, ocasionado pelo acentuado déficit de oxigênio dissolvido [5].

Por outro lado, percebe-se nas campanhas de fevereiro de 2015 e janeiro de 2016, correspondentes ao período seco, que ocorreu uma pequena melhoria na qualidade da água, apresentando resultados do IQAR entre 3 e 3,5, de classificação moderadamente degradado. Neste período, as precipitações mensais nos respectivos meses foram de $97 \mathrm{~mm}$ e $113 \mathrm{~mm}$, diferentemente do período seco de novembro de 2015, marcado pela ausência de precipitação e do mês de outubro de 2016, em que a precipitação mensal foi de $19 \mathrm{~mm}$ [14]. Logo, a precipitação pode ter propiciado a diluição da concentração da matéria orgânica no local, sugerindo poluição de origem antrópica.

O maior valor do IQAR, cujo resultado foi igual a 5,16, registrado no período de baixa precipitação, campanha de fevereiro de 2014, o reservatório obteve a classificação de muito poluído (Classe V). A literatura justifica esse estado pela alta concentração de matéria orgânica, caracterizando como eutrofizado o corpo hídrico [5]. Este resultado negativo pode ser atribuído as elevadas concentrações de nitrogênio total encontradas nas amostras analisadas, demonstrando novamente, a possível entrada de esgoto doméstico no corpo hídrico.

\section{CONCLUSÃO}

O resultado do IQAR possibilitou a identificação de uma forte degradação ambiental no reservatório de Nossa Senhora das Dores, classificando-o como criticamente degradada a poluído, na maioria das campanhas.

Em comparação com legislação pertinente, a Resolução CONAMA 357/2005, os parâmetros encontram-se em desconformidade, ultrapassando assim os limites estabelecidos. Desta forma, o reservatório pertence à classe 4 , cujo corpos hídricos são destinados apenas à navegação e à harmonia paisagística, ou seja, impróprio para o abastecimento público.

Sendo assim, o estudo revela a necessidade de se estabelecer programas de monitoramento da qualidade da água para o controle da poluição, considerando expressivas evidências do elevado aporte de nutrientes e cargas orgânicas nas águas do reservatório.

\section{AGRADECIMENTOS}

Ao Laboratório de Química Ambiental do Departamento de Química da Universidade Federal de Sergipe e ao Instituto Tecnológico e de Pesquisas do Estado de Sergipe - ITPS. E ao Programa de Pós-Graduação em Recursos Hídricos - PRORH.

\section{REFERÊNCIAS BIBLIOGRÁFICAS}

1. Tundisi JG, Straskraba M, Ducan A. Comparative reservoir limnology and water quality management. 1 st ed. České Budějovice: Springer-Science+Business media, BV; 1993. Chapter 13, State-of-the-art of reservoir limnology; p. 213-288, doi:10.1007/978-94-017-1096-1.

2. Brasil. Ministério do Meio Ambiente. Avaliação ambiental integrada de bacia hidrográfica. Brasília: Ministério do Meio Ambiente; 2006. 302 p.

3. Ferreira DM, Cunha C. Simulação numérica do comportamento térmico do reservatório do Rio Verde. Eng Sanit Ambient. 2013 Jan;18(1):83-93, doi: http://dx.doi.org/10.1590/S1413-41522013000100010.

4. Chin DA. Water-quality engineering in natural systems: fate and transport processes in the water environment. New Jersey: WILEY, 2013. 471 p. 
5. Instituto Ambiental do Paraná (IAP). Monitoramento da qualidade da água dos reservatórios. Curitiba, 2014.

6. Secretaria Nacional de Saneamento Ambiental (SNSA). Sistema Nacional de Informações sobre Saneamento: Diagnóstico dos serviços de água e esgotos - 2014. Brasília: SNSA/MCIDADES, 2016. $212 \mathrm{p}$.

7. Superintendência de Recursos Hídricos de Sergipe (SRH). Atlas digital sobre os recursos hídricos de Sergipe. Aracaju, 2016. CD-Rom.

8. Secretaria de Estado do Planejamento e da Ciência e Tecnologia (SEPLANTEC). Atlas digital de recursos hídricos do Estado de Sergipe. Aracaju, 2015.

9. Eaton AD, Rice EW. Standard methods for the examination of water and wastewater. USA: United Book Press; 2012. $541 \mathrm{p}$.

10. Cardoso ETS. Avaliação do grau de trofia e da qualidade da água de um braço do reservatório de ItaipuBrasil [Dissertação]. Santo André (SP): Universidade Federal do ABC; 2011.144 p.

11. Fadini OS, Jardim WF, Guimarães JR. Evaluation of Organic Load Measurement Techniques in a Sewage and Waste Stabilisation Pond. J Braz Chem. 2004;15(1):131-135, doi:http://dx.doi.org/10.1590/S010350532004000100020 .

12. Metcalf L, Eddy HP. Tratamento de efluentes e recuperação de recursos. 5 ed. Porto Alegre: AMGH; 2016. $1980 \mathrm{p}$.

13. Barcella RA. Estudo comparativo entre diferentes técnicas analíticas para determinação da demanda química de oxigênio (dqo) de rios que recebem efluentes [Trabalho de Conclusão de Curso]. Porto Alegre (RS): Universidade Federal do Rio Grande do Sul; 2016. 46 p.

14. Empresa de Desenvolvimento Agropecuário de Sergipe (EMAGGRO). Pesquisa diária de dados pluviométricos. Nossa Senhora das Dores. 2017. 